\title{
FORMACIÓN EN PENSAMIENTO REFLEXIVO Y CRÍTICO EN ENFERMERÍA: ANÁLISIS CURRICULAR EN CUATRO UNIVERSIDADES CHILENAS
}

\section{TRAINING IN REFLECTIVE AND CRITICAL THINKING IN NURSING: CURRICULAR ANALYSIS IN FOUR CHILEAN UNIVERSITIES}

Bruna Espinoza Fernández, Silvana Castillo-Parra, Jessica Olate Andaur, Pamela Torres, Marcela Baeza, Carolina Arévalo Valenzuela y Alejandra Rojas-Rivera

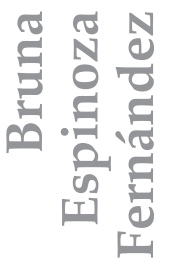

Doctora en Enfermería. Coordinadora Cono-Sur de la Red Iberoamericana de Investigación en Educación en Enfermería. Profesora Adjunta, Universidad de Valparaíso.

bruna.espinoza@uv.cl

Doctora en Educación. Profesora Asociada y Directora del Departamento de Enfermería, Universidad de Chile. Integrante Red Iberoamericana de Investigación en Educación en Enfermería.

scastillop@uchile.cl 


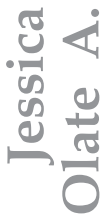

Magister en Educación en Ciencias de la Salud. Profesora Asistente, Universidad de Chile. Integrante de la Red Iberoamericana de Investigación en Educación en Enfermería.

jolate@uchile.cl

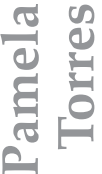

Magister en Educación. Directora Escuela de Enfermería Universidad Diego Portales. Secretaria de la Red Iberoamericana de Investigación en Educación en Enfermería. Región Cono Sur.

Pametorres.p@gmail.com

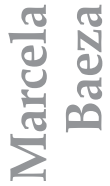

Magister en Docencia para la Educación Superior. Profesora Asistente, Universidad de Santiago. Miembro de la Red Iberoamericana de Educación en Enfermería.

Marcela.baeza@usach.cl

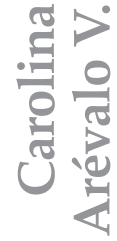

Profesora Asistente, Universidad de Santiago. Miembro de la Red Iberoamericana de Educación en Enfermería.

Carolina.arevalo@usach.cl

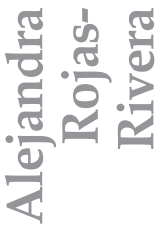

Magister en Enfermería Geriátrica. Profesora Titular, Universidad de los Andes Las Condes, Santiago. Miembro de la Red Iberoamericana de Educación en Enfermería

Enfermería-interalered@gmail.com

\section{RESUMEN}

La formación de enfermeros contempla desarrollar lógicas de razonamiento, para promover el pensamiento reflexivo y crítico (PCR), y aplicarlo en el actuar profesional. Esta investigación pretende determinar 
la presencia de PCR en el currículum de Enfermería de cuatro universidades chilenas, como parte de estudio multicéntrico desarrollado por la Red Iberoamericana de Investigación en Educación en Enfermería (RIIEE) mediante estudio cuantitativo exploratorio-descriptivo. Con aprobación del Comité de Ética, se aplica cuestionario, analizando datos con Programa SPSS. Se caracterizan los docentes de cada unidad académica, número de estudiantes, además de las declaraciones de PCR en Planes de estudio, estrategias didácticas y de evaluación explícitas en los cursos. Concluimos que las cuatro escuelas estudiadas declaran el PRC en sus perfiles y planes de estudio, aunque el nivel de implementación y evaluación en sus cursos es diverso. Futuras investigaciones requieren indagar cualitativamente el modo en que docentes desarrollan PCR con estudiantes, y su impacto en el aprendizaje.

Palabras clave: Educación en Enfermería, pensamiento, currículo,

\section{ABSTRACT}

Nurse training contemplates the development of logic reasoning to promote reflective and critical thinking (RCT) and its application to professional action. The present study aims to determine the presence of RCT in the nursing curriculum of 4 Chilean universities. The multicenter study promoted by the Ibero-American Network of Nursing Education Research (RIIEE) was accomplished by an exploratory-descriptive quantitative study. With the approval of the Ethics Committee, a questionnaire was applied and the data gathered analyzed with the SPSS Program. Descriptions about Professors in each academic unit, number of students, pronouncements of RCT in study plans, didactic strategies and explicit evaluation protocols are provided. Final conclusions point out to the fact that although the four Nursing schools in the study declare RCT in their profiles and study plans, the level of implementation and evaluation in their courses is diverse. Future research requires a qualitative inquiry into the way in which teachers specifically interact with students and their impact on learning.

Keynotes: Education, Nursing, Thinking. 


\section{INTRODUCCIÓN}

La profesión de Enfermería ha ido evolucionado hacia el paradigma de la transformación, que comprende cada fenómeno como único e irrepetible, lo cual inspira el desarrollo de nuevas concepciones de la disciplina enfermera (Martínez, Olivera, 2011). El debate permanente respecto con la concepción de la disciplina de Enfermería, concluye que esta incluiría tanto un cuerpo de conocimientos teóricos, así como también valores, creencias y una práctica compartida entre los miembros de la comunidad de Enfermería (Urra, 2009). En tal contexto, la profesión de Enfermería, conjuntamente con su rol asistencial, orienta la formación de las futuras generaciones en el desarrollo de valores compartidos, además de lógicas de razonamiento, que buscan promover y aplicar el pensamiento reflexivo y crítico en todas las acciones profesionales. Es así que, en las últimas décadas, los términos relacionados con la práctica reflexiva y la reflexión son conceptos que se han incorporado desde etapas iniciales de la formación de los futuros profesionales de Enfermería.

En consideración a lo antes expuesto, parece razonable abordar el concepto de reflexión, cuya complejidad y multidimensionalidad le brindan límites difuminados que obstaculizan definirlo y enseñarlo (Joelle y Kerry 2002), así como consensuar métodos que puedan demostrar el real impacto de sus resultados (Mann, Gordon y MacLeod, 2009). Es innegable el aporte a la conceptualización y aplicabilidad de la reflexión en el aprendizaje y desarrollo intelectual, expuesto entre otros por Dewey (1989), Schön (1992) y Perrenoud (2004). La reflexión es el examen activo, persistente y cuidadoso de toda creencia o supuesta forma de conocimiento a la luz de los fundamentos que la sostienen y las conclusiones a las que tiende (Dewey, 1989). Por su parte, Schön (1992) concibe la reflexión como aprendizaje desde los eventos e incidentes experimentados durante la práctica profesional. Boud et al. (1998), además de considerar los ámbitos intelectuales en la reflexión, incorporan aspectos afectivos que la persona pone en marcha para explorar sus experiencias para alcanzar nuevas apreciaciones y mayor comprensión sobre ellas. Según Somerville (2004), la reflexión como proceso de exploración de la propia experiencia, permite crear y clarificar los propios significados que cada sujeto le otorga a la misma. 
La reflexividad puede resumirse como

[...] un proceso personal de interpretación y comprensión respecto a situaciones problemáticas de la práctica que son siempre únicas y ambiguas, que están saturadas de conflictos de valores, y resultan por tanto inciertas y complejas de enfrentar para el practicante (Castillo, 2014: 66).

Por su parte, Domingo (2012) propone la práctica reflexiva como una metodología de formación donde la experiencia en contexto es el punto de partida para dicha reflexión.

Los trabajos en el ámbito de Enfermería, concluyen que la reflexión ayuda a contextualizar los cuidados enfermeros (Durgahee, 1996) у a integrar la teoría y la práctica (Boud y Walter, 1998). El hábito reflexivo promueve el permanente cuestionamiento de la práctica enfermera, contribuyendo al aprendizaje significativo de los estudiantes de Enfermería y al desarrollo de profesionales expertos (Rodríguez, 2012). Asimismo, la reflexión favorece también la construcción compartida de significados entre los profesionales de Enfermería, lo cual permitiría potenciar la comunicación entre ellos (Medina, 2017).

En este complejo escenario, la Red Iberoamericana de Investigación en Educación en Enfermería (RIIEE), el año 2014, dio inicio a un proyecto multicéntrico titulado «Desarrollo del pensamiento reflexivo y crítico en los estudiantes de Enfermería: situación de Iberoamérica», donde participaron países de Iberoamérica a través de cinco regiones. Dicho estudio posee diversas fases, tanto diagnósticas como de intervención, que apuntan a contar con información sistematizada de evidencias sobre la enseñanza del pensamiento reflexivo, para posteriormente ofrecer, como Red e instancia colegiada, propuestas y recomendaciones de enseñanza aprendizaje, que permitan empoderar a las nuevas generaciones de profesionales de Enfermería, empleando como centro de innovación y desarrollo el pensamiento reflexivo y crítico.

En el contexto antes mencionado, la investigación expuesta en el presente trabajo, corresponde a un estudio de la situación de cuatro universidades chilenas respecto del desarrollo del pensamiento reflexivo y crítico en el currículum de Enfermería. 
Esta investigación aporta conocimientos sobre el contexto de formación en Enfermería, así como la manera de abordar la competencia de pensamiento crítico y reflexivo a lo largo de los cinco años de formación, sus fortalezas y aspectos necesarios de mejorar.

\section{Objetivo General}

Determinar la presencia de la competencia de pensamiento reflexivocrítico o afines en cuatro currículums de Enfermería, chilenos.

\section{Objetivos Específicos}

- Caracterizar cada currículo con base en su referencial pedagógico.

- Identificar tipo de competencias declaradas en las asignaturas del currículo que desarrollan el pensamiento reflexivo y crítico.

- Describir cómo el pensamiento crítico y reflexivo se implementa en estrategias didácticas de las asignaturas que declaran el desarrollo de esta competencia.

- Describir las estrategias de evaluación utilizadas en las asignaturas que desarrollan el pensamiento reflexivo y crítico.

\section{Método}

El enfoque teórico del estudio es el paradigma cuantitativo, transversal, exploratorio y descriptivo.

\section{Población}

La población objeto de estudio corresponde a los currículos de los programas de estudio de las Escuelas de Enfermería, pertenecientes a la Asociación Chilena de Escuelas de Enfermería (ACHIEEN). N= 21 


\section{Muestra}

Cada directora o responsable de los planes de estudio de las universidades que cumplían con los criterios de inclusión, fue invitada a participar del estudio, mediante presentación del proyecto en una reunión del Consejo Directivo de ACHIEEN. Solo cuatro universidades acreditadas para impartir la carrera de Enfermería en Chile (dos públicas y dos privadas), autorizaron por escrito participar en el estudio. De acuerdo con lo anterior, se optó por un muestreo por conveniencia de una muestra que incluyó los cuatro currículum de Enfermería de dichas universidades.

\section{Criterios de Inclusión}

- Currículum de unidades académicas que pertenecieran a la Asociación Chilena de Escuelas de Enfermería (ACHIEEN) y estuviesen, por tanto, acreditadas a nivel nacional.

\section{Variables Estudiadas}

1. Caracterización de la unidad académica. Perfil del cuerpo docente que se desempeña en cada unidad académica de Enfermería, cuantificando elementos como: número de contratos de profesionales de Enfermería, jornadas equivalentes, niveles y grados de formación de las académicas; además, considera el número de estudiantes matriculados por cada nivel de estudios en la respectiva Escuela de Enfermería.

2. Referencial pedagógico. Compromiso formativo declarado por la institución participante en su programa de estudio.

3. Presencia de la competencia de pensamiento reflexivo-crítico o similar, en el currículum. Declaración explícita de competencias en el currículo que promuevan el desarrollo del pensamiento crítico y reflexivo y afines, en los estudiantes. 
4. Metodologías docentes empleadas para trabajar la competencia en estudio. Declaración explícita de contenidos y estrategias didácticas en los programas de cada curso del currículo, que se relacionan con el desarrollo del pensamiento crítico y reflexivo.

5. Proceso de evaluación de la competencia. Estrategias de evaluación declaradas en cada curso del currículo, relacionadas con el pensamiento crítico y reflexivo.

\section{Instrumentos de Recogida de Datos}

Los datos fueron recolectados a través de un cuestionario confeccionado ad hoc por miembros de RIIEE, investigadores y coinvestigadores de las regiones México, Brasil, Región Andina y Europa. Se aplicó un mismo instrumento para la recolección de datos en todos los países miembros.

Para la presente investigación, el cuestionario fue aplicado por un coinvestigador miembro de RIIEE a un docente encargado del programa de Enfermería de cada unidad académica que aceptó participar en esta investigación. El investigador envió por correo el cuestionario y coordinó una fecha para una entrevista y entrega del cuestionario completo.

El cuestionario contó con 38 preguntas distribuidas en los siguientes ítems:

- Ítem I. Datos generales de la unidad académica participante: preguntas 1-9.

- Ítem II. Datos de la persona que respondió el cuestionario, docente encargado del programa de estudio; director(a) de la unidad académica o jefe(a) de carrera. Pregunta: 10-11.

- Ítem III. Caracterización de los docentes de la carrera de Enfermería. Preguntas 12-17. 
- Ítem IV. Aspectos generales del currículo. Preguntas: 18-29.

- Ítem V. Evidencia de pensamiento crítico y reflexivo en el currículo. Preguntas: 30-38.

\section{Análisis de Datos}

Los datos se recogieron en una planilla Excel. Se confeccionó una base de datos y luego fueron traspasados y analizados en el sistema computacional SPSS versión 21.

\section{Aspectos Éticos del Estudio}

Con el objeto de anonimizar la muestra se asignó la siguiente denominación a cada una de las universidades participantes U1-U2 - U3- U4.

Las unidades académicas que participaron en el estudio firmaron un consentimiento informado.

Este estudio cuenta con autorización del Comité de Ética de la Facultad de Medicina de la Universidad de Chile, archivo 140/2016.

\section{RESULTADOS}

A continuación, se darán a conocer los aspectos más relevantes que dan respuesta a los objetivos de estudio.

\section{Caracterización de la Planta de Docentes y Estudiantes Matriculados en las Instituciones Participantes}

Las unidades académicas participantes poseen un número de docentes muy diverso, con distinto nivel de formación también. Como base, todos con grado de licenciatura, requisito para ser contratados; un número importante con grado de magister o maestría y un número menor con grados de doctorado. En el gráfico 1, la unidad U1 es la que 
posee un 35\% de académicos con grado doctorado, seguido de la U4 que muestra un $12 \%$, mientras que U2 muestra un $10 \%$ y por último U3 un $2 \%$. Cabe destacar que en Chile es escasa la oferta de programas de formación doctoral en Enfermería, por lo cual la mayoría de los profesionales deben cursar programas fuera del país con dedicación parcial de estudios.

Las jornadas completas equivalentes (JCE) corresponden a la cantidad de horas totales contratadas de académicos en una unidad, que son divididas por una jornada completa. En Chile, la jornada completa de trabajo es de 44 horas / semana. Las unidades participantes fluctúan sus jornadas completas de contrato entre 9,75 y $53 \mathrm{JCE}$, con una distribución de estudiantes de acuerdo a la tabla 1.

\section{Gráfico 1. Perfil docente de las unidades académicas participantes.}

\section{PERFIL DOCENTE}

$\square \mathrm{U} 1 \square \mathrm{U} 2 \square \mathrm{U}$ 3 $\square 4$

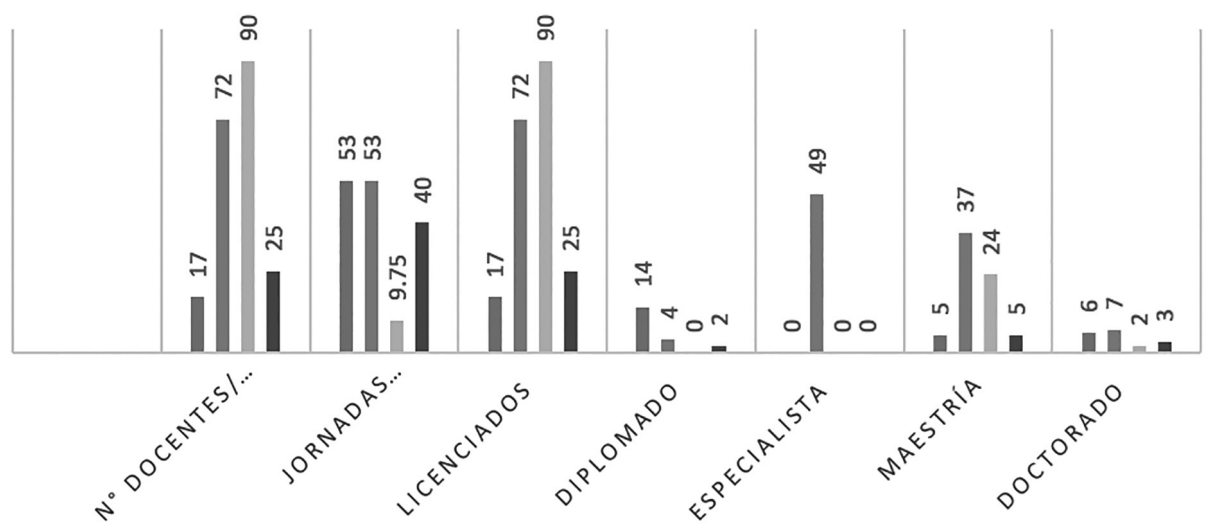

Fuente: Base de datos propios. 
Tabla 1. Relación estudiante, número de jornadas equivalentes contratadas por unidad académica.

\begin{tabular}{cccc}
\hline UNIDAD ACADEMICA & $\begin{array}{c}\text { N } \text { JCE } \\
\text { CONTRATADOS }\end{array}$ & $\begin{array}{c}\mathrm{N}^{\circ} \text { TOTAL } \\
\text { ESTUDIANTES }\end{array}$ & $\begin{array}{c}\text { RELACION } \\
\text { ESTUDIANTES/ } \\
\text { DOCENTES }\end{array}$ \\
\hline UA 1 & 53 & 431 & 8.1 \\
\hline UA 2 & 53 & 502 & 9.47 \\
\hline UA 3 & 9.75 & 558 & 57.23 \\
\hline UA 4 & 40 & 288 & 7.2
\end{tabular}

Fuente: Base de datos propios.

\section{Caracterización del Currículo}

Las unidades académicas se adscribieron a más de un modelo pedagógico; solo U1 evidencia un modelo por competencias. Se destaca que en todas las universidades, el modelo pedagógico dominante es el centrado en el estudiante. Ver gráfico:

\section{Gráfico 2. Referencial pedagógico del currículo.}

\section{Referencial Pedagógico del currículo}

Metodologías con TICS Métodos participativos

Métodos Mixtos

Método Tradicional

Centrado en el alumno Basado en Competencia

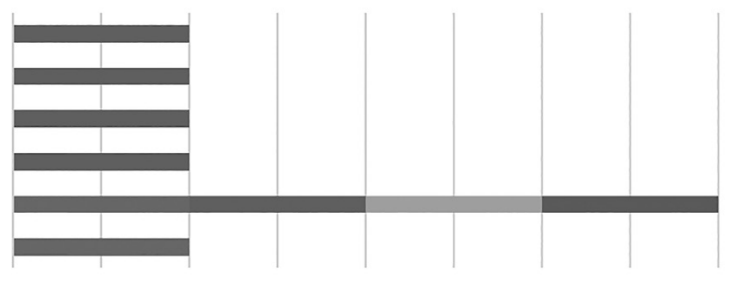

$\begin{array}{llllllllll}0 & 0.5 & 1 & 1.5 & 2 & 2.5 & 3 & 3.5 & 4 & 4.5\end{array}$

$\square \mathrm{U} 1 \square \mathrm{U} 2 \square \mathrm{U} 3 \square \mathrm{U} 4$

Fuente: Base de datos propios. 


\section{Competencias Declaradas en las Diferentes Asignaturas del Currículo}

De acuerdo con los resultados obtenidos, se aprecia una gran diversidad de competencias adscritas a las diferentes asignaturas de los currículos estudiados.

Ninguna unidad académica coincide en forma textual en la redacción; sin embargo, todas apuntan a desarrollar en los estudiantes un pensamiento crítico y reflexivo.

A continuación, se describen algunas de las competencias declaradas en los programas de asignatura por las instituciones participantes (tablas 2 a 5).

Tabla 2. Resumen de competencias declaradas en los programas de cursos. Unidad Académica U1.

\section{COMPETENCIAS}

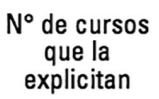

Propone juicios reflexivos mediante el análisis de concepto, proceso y resultados estableciendo acciones en diferentes contextos.

Evidencia conductas sujetas a valores, enfoque pluralista y pensamiento reflexivo.

Resuelve pertinentemente situaciones imprevistas y complejas, aplicando el pensamiento crítico y reflexivo, lo que permite actuar en diferentes contextos.

Argumenta desde el rol profesional su compromiso ciudadano. 1 Indaga e identifica situaciones derivadas de la práctica de Enfermería, 3 utilizando el pensamiento crítico y reflexivo en la búsqueda de la evidencia, en las diferentes áreas del desempeño.

Desarrolla el pensamiento crítico, mediante la búsqueda de evidencias que expliquen los fenómenos observados en las diferentes áreas del desempeño.

Fuente: Base de datos propios. 


\section{Tabla 3. Resumen de competencias declaradas en} los programas de asignaturas. Unidad Académica U2.

\section{COMPETENCIAS}
$\mathrm{N}^{\circ}$ de cursos
que la
explicitan

Reflexiona acerca del fin del hombre y de aquello que, en cuanto ser libre, lo conduce 0 aparta de él.

Resuelve problemas con autonomía, utilizando el pensamiento crítico.

Usa del pensamiento crítico en relación a la literatura científica.

\section{1}

1

Fuente: Base de datos propios.

\section{Tabla 4. Resumen de competencias declaradas en los programas de asignaturas. Unidad Académica U3.}

\section{COMPETENCIAS}

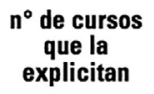

1

Identificar estrategias que favorezcan el pensamiento crítico, trabajo en equipo y habilidades de comunicación.

Demostrar principios, valores éticos y pensamiento crítico en el quehacer de la gestión del cuidado.

Utiliza el conocimiento y el razonamiento clínico para el cuidado de las personas.

Emplea el pensamiento reflexivo para la toma de decisiones en la gestión del cuidado del adulto hospitalizado.

Comprende el desarrollo del pensamiento científico y el razonamiento inductivo.

Adopta juicios clínicos y toma decisiones en el contexto de la 1 gestión del cuidado de las personas mayores.

Lectura crítica que identifique elementos de rigurosidad metodológica y transferibilidad de los resultados.

Resuelve en forma reflexiva y responsable los problemas de salud 2 que afectan a la persona, familia y comunidad.

Desarrolla un pensamiento crítico, creativo y metacognitivo para la elaboración de un proyecto de investigación en Enfermería.

Fuente: Base de datos propios. 
Tabla 5. Resumen de competencias declaradas en los programas de asignaturas. Unidad Académica U4.

\section{COMPETENCIAS}

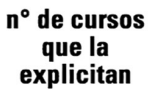

Incentiva la capacidad reflexiva.

Desarrolla la capacidad de conceptualización, análisis, síntesis y evaluación de la información generada por la observación, la experiencia, la reflexión y el razonamiento.

Aplica conocimientos teóricos en el tratamiento farmacológico de un paciente, reconociendo críticamente las reacciones adversas e interacciones farmacológicas.

Fuente: Base de datos propios.

\section{Cómo se Desarrolla en los Cursos el Pensamiento Crítico y Reflexivo}

El pensamiento crítico y reflexivo, se inserta principalmente en el proceso de evaluación y a través de las estrategias didácticas, siendo menos desarrollado a nivel de los contenidos:

Gráfico 3. Número de asignaturas que evidencian la competencia pensamiento crítico y reflexivo en el currículo de las Unidades Académicas participantes.

\section{Competencia pensamiento reflexivo-crítico o afines en contenidos, estrategias didácticas y evaluación por Unidad Académica.}

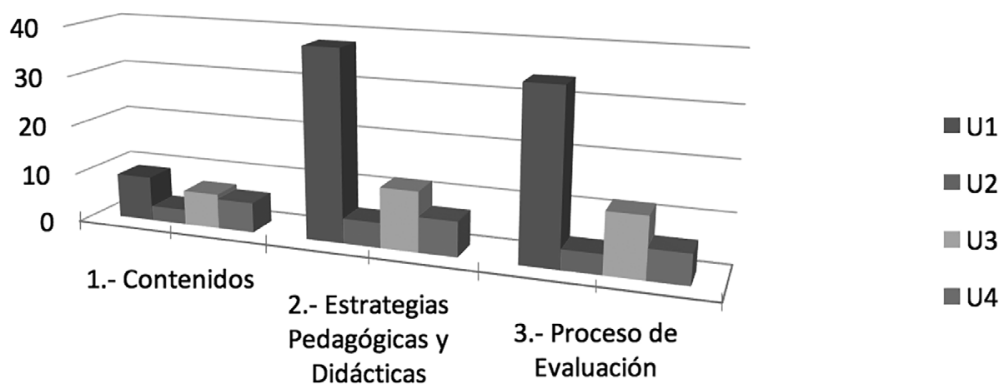

Fuente: Base de datos propios. 


\section{Estrategias de Evaluación del Aprendizaje Utilizadas en las Asignaturas}

En todas las universidades existen diversas estrategias de evaluación, coherentes con el pensamiento crítico y reflexivo. Entre las más empleadas se destacan: autoevaluación por pares, rúbricas, pruebas prácticas, ensayo teórico-práctico OSCE, simulación clínica, entre otras. Entre las menos utilizadas se destacan el diario reflexivo, el método de resolución de problemas y las pruebas en línea. A continuación, se exponen las estrategias de evaluación utilizadas:

\section{Tabla 6. Estrategias de Evaluación utilizadas por las unidades académicas participantes.}

\begin{tabular}{ll} 
1. Autoevaluación por pares & 10. Ensayos seminario \\
\hline 2. Rúbricas & 11. Simulación clínica \\
3. Pruebas prácticas & 12. Ensayo-seminario \\
4. Ensayo teórico-práctico & 13. Análisis crítico documentos \\
5. Portafolio-bitácora & 14. ABP \\
6. Informe escrito & 15. Virtual \\
7.-OSCE & 16. Web 2.0 \\
8. Diario reflexivo & 17. Foro \\
9. Pruebas línea & 18. Trabajo colaborativo
\end{tabular}

Fuente: Base de datos propios.

\section{DISCUSIÓN}

Se destaca que en todas las universidades, el modelo pedagógico es centrado en el estudiante, lo cual orienta a una formación para el desarrollo de competencias diversas —desde los ámbitos académicos, personales, sociales y culturales-, respetando la identidad de las personas que conforman la comunidad universitaria. Si bien el modelo pedagógico se determina por la institución, son los docentes quienes materializan esto a nivel de los procesos de enseñanza-aprendizaje en los distintos escenarios y en el diseño 
de programas de asignaturas para el cumplimiento curricular. Por lo anterior, es relevante explicitar que las concepciones que tienen los docentes y el significado que le asignan al proceso de enseñanza-aprendizaje, desde su propia experiencia y conocimiento, intencionan estos procesos que en ocasiones no necesariamente están alineados con los modelos pedagógicos declarados institucionalmente. Es indispensable recapacitar para resignificar la práctica reflexiva y dar coherencia a la formación profesional (García, Forero, Ocampo y Madrigal, 2015).

Esta coherencia se asocia a la versatilidad del mundo actual, que requiere de profesionales de Enfermería más críticos, creativos y vinculados a la realidad, con poder de adaptación a las demandas socioculturales, políticas de salud y temáticas emergentes, a nivel nacional e internacional.

Con base en lo anterior, esto exige a los centros formadores la realización de modificaciones curriculares con una efectiva reorientación y / o transformación de la docencia (Do Carmo et al., 2013). Siendo fundamental contar con docentes capaces de potenciar el pensamiento crítico para la toma de decisiones, desarrollo de autonomía y racionalidad, con el fin de mejorar las prácticas no solo en el área profesional, sino también en la disciplinar (Demandes e Infante, 2017).

La definición de pensamiento crítico sigue siendo materia de análisis, pero se puede entender como el

[...] proceso cognitivo racional de orden superior, desarrollado a partir de las tendencias a pensar en una cierta forma y a hacer algo en determinadas condiciones, que integra el conocimiento conceptual con la experiencia mediante la problematización contextualizada, conducente a la construcción de hipótesis sustentadas en evidencias para lograr juicios. Este pensamiento implica inferencia, evaluación, argumentación, análisis e interpretación (Ospina, Brand y Aristizábal, 2017).

Contexto en el cual las competencias declaradas en los programas curriculares hacen referencia a estos múltiples elementos que enmarcan este complejo proceso cognitivo a lo largo de la formación de los profesionales de Enfermería, pero a su vez necesitan lograr coherencia 
micro-curricular a nivel de los contenidos y las metodologías docentes formativas y evaluativas.

En el estudio, el pensamiento crítico y reflexivo se inserta principalmente en los procesos de evaluación y a través de las estrategias didácticas, pero es menos desarrollado a nivel de contenidos, lo cual pudiera limitar el anclaje y la aplicación fundamentada por parte de los profesionales en formación. En este sentido se visualiza la relevancia de desarrollar estrategias cognitivas y metacognitivas, encaminadas a adquirir herramientas para el aprendizaje autorregulado que facilitaría el éxito en diferentes áreas no solo académicas, sino también personales y socioculturales, que es el propósito del modelo pedagógico centrado en el estudiante (Márquez, Andrade y Cuevas, 2017).

Dentro de las diversas estrategias empleadas en los programas curriculares, se visualizan metodologías activas e innovadoras, principalmente para el proceso de evaluación coherentes con el pensamiento crítico y reflexivo, las cuales deberían ser conocidas y fundamentadas de manera formal, teniendo presente la individualidad de cada estudiante, facilitando así el desarrollo y progreso de sus potencialidades a través de su formación en las diversas áreas (Bernal y Martínez, 2009).

Estos resultados permiten tener una visión de la situación actual y definir mejoras curriculares, enfocadas a promover el desarrollo del pensamiento crítico de estudiantes y docentes en aspectos de carácter científico, técnico, humano y social, guiado por la motivación de la «construcción de horizonte del cuidado, y una experiencia de enseñanza-aprendizaje significativa para el profesional de Enfermería en el contexto social». Para lo cual es prioritario estandarizar la medición, diseño y evaluación del pensamiento crítico en la formación de Enfermería, pues la práctica profesional y disciplinar

[...] debe estar orientada por un proceso educativo que tenga la intencionalidad de favorecer el desarrollo de habilidades cognitivas y actitudinales para la indagación, la problematización, la identificación y el análisis de las necesidades de cuidado, su interpretación y argumentación, que dé sentido a las prácticas de cuidado desde lo profesional y disciplinar, tanto para el sujeto cuidado como para la sociedad (Ospina et al., 2017). 
En conclusión, el estudio permite analizar las estrategias empleadas para fomentar el desarrollo de la competencia de pensamiento crítico en estudiantes de Enfermería, las fortalezas y los aspectos por mejorar que facilitaran el diseño de propuestas para avanzar en el proceso de empoderar a las nuevas generaciones de profesionales de Enfermería, empleando como centro de innovación y desarrollo el pensamiento reflexivo y crítico.

\section{REFERENCIAS}

Bernal, M., Martínez, M. (2009). Metodologías activas para la enseñanza y el aprendizaje. Revista Panamericana de Pedagogía Saberes y Quehaceres del Pedagogo, 14, 101-106.

Boud, D. \& Walker, D. (1998). «Promoting reflection in professional courses: the challenge of context». In Studies in Higher Education. 23(2), 191-206.

Castillo, S. (2014). Práctica pedagógica de profesores/as expertos/as en la formación clínica de estudiantes de Enfermería. Tesis doctoral. Universitat de Barcelona.

Demandes, I., Infante, A. (2017). «Desarrollo del pensamiento crítico en la formación del profesional enfermero». Ciencia y Enfermería, 23(2), 9-12. Recuperado de https://dx.doi.org/10.4067/S0717$\underline{95532017000200009}$

Dewey, J. (1989). Cómo pensamos. Nueva exposición de la relación entre pensamiento reflexivo y proceso educativo. Barcelona: Paidós.

Domingo, A. (2012). ¿Qué es la práctica reflexiva? https://edu21.cat/ Disponible en: http://campus.usal.es/ magisterioza/secretaria docs $/$ practicum $/ \mathrm{Qu} \% \mathrm{C} 3 \% \mathrm{~A} 9 \% 20 \mathrm{es} \% 20 \mathrm{la} \% 20 \mathrm{Pr} \% \mathrm{C} 3 \% \mathrm{~A} 1 \mathrm{ctica} \% 20$ reflexiva.pdf

Do Carmo, J; Kloh, D; Mattioli, H., Gue, J; de Souza, F. \& Schubert, V. (2013). «Reliving the debate concerning nursing education: from where we departed and to where we are going». Investigación y Educación en Enfermería, 31(3), 395-405. 
Durgahee, T. (1996). «Promoting reflection in postgraduate nursing: a theoretical model». Nurse Education Today. 16, 419-426.

García, L., Forero, C., Ocampo, D., Madrigal, M. (2015). «El significado de enseñar y aprender para los docentes». Investigación y Educación en Enfermería, 33(1), 8-16.

Jiménez, M. A. y Col. (2012). Desarrollo del Pensamiento Reflexivo y Crítico en los estudiantes de Enfermería: situación de Iberoamérica Red Iberoamericana de Investigación en Educación en Enfermería. Disponible en: https:// riiee.jimdo.com/investigaci\%C3\%B3n/

Joelle, K. Jay. \& Kerry, L. Johnson (2002). «Capturing complexity: a typology of reflective practice for teacher education». Teaching and Teacher Education. 18, 73-85.

Mann, K., Gordon, J., \& MacLeod, A. (2009). Reflection and reflective practice in health professions education: a systematic review. Advances in health science education: theory and practice, 14(4), 595-621.

Márquez, N., Andrade, A. y Cuevas, J. (2017). «Estrategias cognitivas y metacognitivas en estudiantes de educación secundaria con aptitudes sobresalientes». Revista Panamericana de Pedagogía Saberes y Quehaceres del Pedagogo. 24, 115-133.

Martínez, L. y Olvera, G. (2011). «El paradigma de la transformación en el actuar de Enfermería». Revista de Enfermería del Instituto Mexicano del Seguro Social. 19 (2): 105-108.

Medina, J. L. (2017). La pedagogía del cuidado. Saberes y prácticas de la formación de profesionales de Enfermería. Santiago: Editorial Universitaria.

Ospina, B., Brand, E. y Aristizabal, C. (2017). «Desarrollo de un índice de medición del pensamiento crítico en la formación profesional». Investigación y Educación en Enfermería, 35(1), 69-77. Recuperado de: https:/ / dx.doi.org/10.17533/udea.iee.v35n1a08

Perrenoud, P. (2004). Desarrollar la práctica reflexiva en el oficio de enseñar. Barcelona: Graó.

Rodríguez, M. (2012). El arte del cuidado: Un legado reflexivo de saber. Tesis Doctoral. Universidad Europea de Madrid. 
Schön, D. (1992). La formación de profesionales reflexivos: Hacia un nuevo diseño de la enseñanza y el aprendizaje en las profesiones. ( $2^{\circ}$ ed.). Barcelona: Paidós.

Somerville, D. \& Keeling, J. (2004). «A practical approach to promote reflective practice within nursing». Nursing Times. 100(12), 42-5.

Urra, E. (2009). «Avances de la ciencia de Enfermería y su relación con la disciplina». Ciencia y Enfermería, 15(2), 9-18. Recuperado de: https:// dx.doi.org/10.4067/S0717-95532009000200002 